\title{
ORIGINAL ARTICLE Clinical analysis following lumbar interspinous devices implant: where we are and where we go
}

\author{
G Grasso $^{1}$, F Giambartino ${ }^{2}$ and DG Iacopino ${ }^{1}$
}

\begin{abstract}
Study design: Retrospective study.
Objectives: We present our experience with patients treated with interspinous devices who are affected by neurogenic intermittent claudication (NIC) or lumbar disc herniation (LDH) where the interspinous system has been inserted following microdiscectomy. Methods: This study included patients $(n=100)$ with NIC secondary to lumbar spinal stenosis (group 1$)$, and patients $(n=100)$ with LDH (group 2) in whom the interspinous device has been implanted following radicular decompression in a period spanning 6 years. The latter have been compared with a homogenous group of patients $(n=100)$ where no interspinous system has been implanted following microdiscectomy (group 3). Clinical findings have been observed preoperatively and 3, 6, 12 months and every year postoperatively using dedicated questionnaires (Zurich Claudication Questionnaire, Visual Analog Scale and Oswestry Disability Index). Results: Six years following surgical treatment, $85 \%$ of the patients of group 1 presented good improvement of symptoms and $90 \%$ of the patients referred satisfaction for surgery. Only few cases needed reoperation. In one case, the device was removed and in two cases, we changed the surgical strategy. Overall, patients of group 2 presented significantly less lumbar disc recurrences compared with group $3(P<0.05)$ and better clinical outcome when compared with the same group $(P<0.01)$.

Conclusion: According to our features, interspinous systems showed significant and clinically meaningful improvements in pain and disability for up to 6 years. Furthermore, interspinous devices have shown better clinical outcome and less lumbar disc recurrences when associated with standard microdiscectomy. These data, however, need further studies and a longer period of follow-up.
\end{abstract}

Spinal Cord (2014) 52, 740-743; doi:10.1038/sc.2014.100; published online 10 June 2014

\section{INTRODUCTION}

Lumbar spinal stenosis (LSS) is a result of degenerative, developmental or congenital disorders. The degenerative type occurs most often, especially in those 50-60 years of age. ${ }^{1}$ People with the congenital type may complain earlier in life; their stenosis is a result of congenitally anatomic changes or malformations. Developmental spinal stenosis is a condition in which the narrow spinal canal is caused by a growth disturbance of the posterior elements in the spinal canal.

LSS may occur at different localizations in the spinal canal. In central canal stenosis, nerve roots and the cauda equina may be compressed. Lateral recess stenosis and foraminal stenosis may cause compression of the nerve roots leaving the spine. Symptoms of spinal stenosis include lower back pain as well as unilateral or bilateral groin and leg pain, numbness or weakness. Neurogenic claudication, defined as pain, paresthesia and cramping of one or both lower extremities due to neurologic compromise brought on by standing or walking and relieved by sitting, is the most specific symptom of spinal stenosis.

Advances in imaging technology have led to a marked increase in the diagnosis of spinal stenosis, and spinal stenosis is now the most common diagnosis among those having lumbar spine surgery. Despite the common nature of the condition and the widespread acceptance in clinical practice of various surgical and nonsurgical interventions for individuals with symptomatic spinal stenosis, there is limited evidence to support many of them, especially in terms of their relative benefit and risk compared with other options. The optimum treatment of LSS is generally considered to be surgical intervention, as two randomized clinical trials comparing conservative treatment with conventional bony decompression resulted in treatment effects in favor of surgery. ${ }^{2,3}$ Considering the destructive nature of bony decompressive surgery of the spinal column when performing lumbar spine laminectomy, ${ }^{4}$ the resulting instability often requires subsequent instrumental spondylodesis. ${ }^{5}$

In this scenario, several devices designed for minimally invasive posterior stabilization have been introduced. These devices can be divided into three fundamentally different constructs: (1) facet replacement; (2) transpedicular insertion semirigid rods; and (3) interspinous process stabilization devices. Under the subheading of interspinous process stabilization devices, there are two types: soft and rigid. In theory, these devices are expected to achieve local kyphosis, improve sagittal balance, provide segmental stability, modify posterior neural foraminal height, provide pain relief, particularly in patients suffering from low back pain due to facet overloading or segmental instability or increase lumbar spinal canal diameter in patients affected by LSS. Furthermore, due to their segmental properties, it has been thought to have a role in maintaining lumbar intervertebral height following discectomy.

${ }^{1}$ Section of Neurosurgery, Department of Experimental Biomedicine and Clinical Neurosciences (BIONEC), University of Palermo, Palermo, Italy and ${ }^{2}$ Department of Anesthesiology, University of Palermo, Palermo, Italy

Correspondence: Professor G Grasso, Neurosurgical Clinic, Department of Experimental Biomedicine and Clinical Neurosciences, University of Palermo, School of Medicine, Via del Vespro 129, 90100 Palermo, Italy.

E-mail: giovanni.grasso@unipa.it

Received 3 March 2014; revised 1 May 2014; accepted 11 May 2014; published online 10 June 2014 
In this study of retrospectively reviewed patient data collected over 6 years, we have attempted to verify the properties of interspinous devices in patients with LLS and lumbar disc herniation (LDH) in whom the interspinous system has been inserted following microdiscectomy.

\section{PATIENTS AND METHODS}

In this study, 100 patients with neurogenic intermittent claudication secondary to LSS (group 1) and 100 patients with LDH (group 2) in whom the interspinous device has been implanted following radicular decompression were investigated in a period from March 2006 to January 2013. Group 2 has been compared with a homogenous cohort of patients $(n=100)$ where no interspinous system has been implanted following microdiscectomy (group 3 ). Table 1 shows the demographic data for of all the patients.

For patients of group 1, inclusion criteria were age $\geqslant 45$ years, persistent leg, buttock, or groin pain, with or without back pain, which was relieved by lumbar flexion, symptomatic and undergoing unsuccessful conservative treatment for at least 6 months, diagnosis of moderate LSS (both central and lateral), defined as $25-50 \%$ reduction in lateral/lumbar spinal canal diameter compared with adjacent levels and radiographic evidence of thecal sac and/or cauda equine compression, nerve root impingement by either osseous or non-osseous elements and/or hypertrophic facets with canal encroachment.

Exclusion criteria included LSS at three or more levels, grade II to V spondylolisthesis, significant lumbar instability, important systemic diseases, vertebral osteoporosis or history of vertebral fracture.

Among patients affected by LSS, 50 patients were implanted with X STOP interspinous device (Medtronic, Inc., Sunnyvale, CA, USA), and 50 with Impala (SIGNUS Medizintechnik GmbH, Germany). The first is a titanium implant that consists of two components: a spacer assembly and a wing assembly. The second is made from PEEK and features a modulus that is in the range of cortical bone. The implant consists of two wings that are spread in order to fix the position. A plate is utilized to lock this construct. For X-ray, the implant features tantalum markers. For both the above-mentioned devices, five sizes are available, ranging from $8-16 \mathrm{~mm}$, with the sizes corresponding to the amount of desired distraction between the two spinous processes.

Group 2 patients were classified as suitable candidates for microdiscectomy and interspinous system placement, for which the reasoning was the stabilization of the intervertebral segment considering on preoperative studies considerable disc material removal, thus preventing disc height collapse; prevention of further segmental degeneration, facet disease and consequent adjacent-segment disease. In this group of patients, 40 impala and 60 XSTOP were implanted, respectively.

For all patients, medical history was carefully investigated, physical examination along with neurological evaluation was achieved. X-rays (standing $\mathrm{A} / \mathrm{P}$, lateral lumbar, flexion/extension lateral lumbar) and magnetic resonance

\section{Table 1 Demographic data in all the groups}

\begin{tabular}{|c|c|c|c|}
\hline \multirow[t]{2}{*}{ Characteristic } & \multicolumn{3}{|c|}{ Value } \\
\hline & Group 1 & Group 2 & Group 3 \\
\hline Number & 100 & 100 & 100 \\
\hline \multicolumn{4}{|l|}{ Sex } \\
\hline Male & 47 & 51 & 46 \\
\hline Female & 53 & 49 & 54 \\
\hline \multicolumn{4}{|l|}{ Age (years) } \\
\hline Mean \pm s.d. & $65.3 \pm 5.2^{\mathrm{a}}$ & $50.61 \pm 7.4$ & $52.3 \pm 4.8$ \\
\hline Range & $48-78$ & 29-73 & $25-75$ \\
\hline \multicolumn{4}{|l|}{$B M I$} \\
\hline Mean \pm s.d. & $25.2 \pm 2.4$ & $26.1 \pm 3.12$ & $28.5 \pm 3.53$ \\
\hline Range & $18-32.5$ & $18.4-33.1$ & $18.7-34.9$ \\
\hline
\end{tabular}

Abbreviation: BMI, body mass index.

aStatistically significant difference compared with the other groups $(P<0.05)$ imaging or computed tomography of the lumbar spine was performed in all the cases. The Zurich Claudication Questionnaire (ZCQ) was utilized to assess patient-reported measures of symptom severity, physical function and patient satisfaction. Extremity and axial pain severity were measured with a $100 \mathrm{~mm}$ visual analog scale. Degree of back-specific functional disability was assessed with the Oswestry Disability Index (ODI).

Patients were operated on while under general or local anesthesia in a prone position and received an antibiotic prophylaxis before the surgery. Local anesthesia was used for patients of group 1.

Briefly, a midline skin incision of approximately $3-3.5 \mathrm{~cm}$ was made above the spinous processes of the stenotic level. The paraspinal muscle was elevated from both sides (one side when Impala was implanted) of the spinous processes to the level of the facets and laminae. The supraspinous ligament was preserved and a curved dilator was used to pierce the interspinous ligament and locate the space between the spinous processes.

Under fluoroscopy, the operative level was verified and the interspinous space was gently sized with a sizing distractor. The correct implant size was determined by opening the sizing distractor until the supraspinous ligament was taut. The fluoroscopy showed the opening of the selected space. The interspinous system was inserted, generally from the right side, as close to the laminae as possible. Finally, the wound was closed and patients were allowed to get up at the first postoperative day.

In case of implant following microdiscectomy, the above-mentioned procedure was performed following disc removal, under general anesthesia.

\section{Clinical outcome measurement}

We observed clinical findings preoperatively and 3, 6, 12 months and every year post-operatively using dedicated questionnaires. The Visual Analog Scale (VAS), ODI and ZCQ patient assessment scales were used to evaluate the outcome in this study. These assessments are reported for baseline and at 12 weeks, 6 months and every year post-operatively. The VAS provides a numerical measurement of back and leg pain intensity on a 10-point continuum, with 1 denoting no pain and 10 indicating the worst pain possible. The ODI provides a measurement of functional disability resulting from chronic back pain. ODI scores range from zero to 100 , with higher scores signifying greater disability. The ZCQ is a validated patient-reported outcomes tool. ZCQ consists of symptom severity and physical function domains that are recorded at baseline and at each follow-up interval. In addition, ZCQ also contains a Patient Satisfaction Domain that is completed only at follow-up. For each ZCQ domain, higher scores indicate worse patient condition. As a validated patient outcome tool specific to LSS, ZCQ provides information specifically related to spinal disability.

\section{Statistical analysis}

Data were reported as mean \pm s.d. and categorical data were reported as frequencies and percentages. The clinical results were analyzed using the analysis of variance $\chi^{2}$-square test, Fisher exact test, Kruskal-Wallis test and McNemar test.

\section{RESULTS}

Patients were compared in terms of sex, symptoms, age and body mass index. Demographic differences among the groups were not statistically significant except for age being statistically higher in group 1 compared with the other groups $(P<0.05)$.

A total of 114 interspinous systems were inserted in group 1 . The most common level of insertion was L4-L5. A mean of 1.1 interspinous devices were inserted in each patient (range 1-2; seven patients had two implants). The most common device size used was $10 \mathrm{~mm}$.

In group 1 , in three cases, the implant had been changed by a reoperation. In one case for implant dislocation, in two cases the device was removed as it was not tolerated by the patient and different surgical procedure was performed. In group 2, all the devices were well tolerated without the need for further surgical treatments. No significant differences have been observed between the two devices used. No infections were observed in all the patients. 

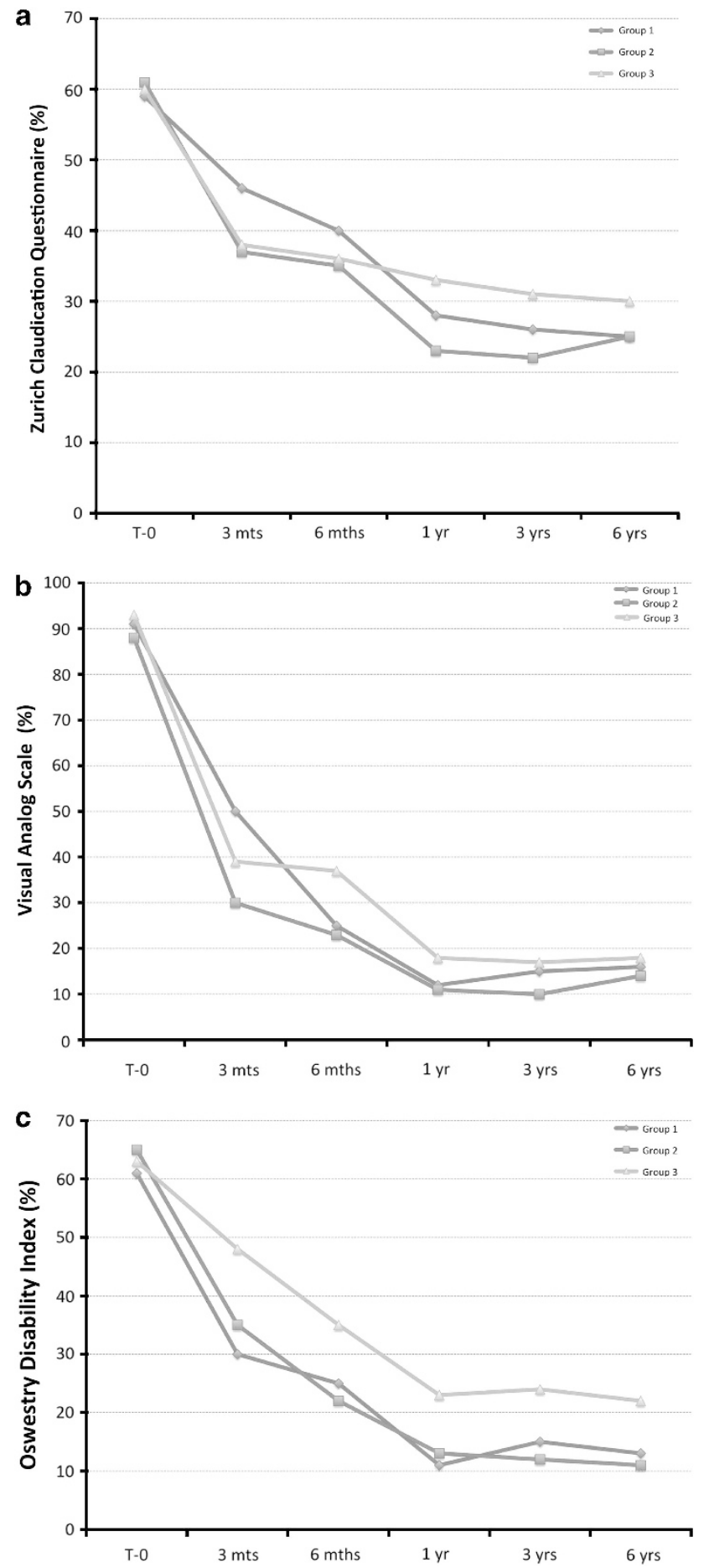

Figure 1 Bar graphs showing preoperative and postoperative ZCQ (a), VAS (b) and ODI (c) outcomes among the groups. Overall, ZCQ, VAS and ODI score improved in all the groups at 1-year follow-up without significant variation in the subsequent follow-up. Significant statistical differences were noted in all the groups when comparing the clinical outcome measures from baseline to 1-year follow-up. No statistically significant differences were noted between group 1 and 2. A better clinical outcome was observed in group 2 when compared with group $3(P<0.01)$.

Six years following surgical treatment, $85 \%$ of the patients of group 1 presented with a very good improvement of symptoms and $90 \%$ of patients referred satisfaction for surgery. Overall, the patients of group
Table 2 Mean preoperative and postoperative ZCQ, VAS and ODI scores for all the groups

\begin{tabular}{llllllll}
\hline & \multicolumn{3}{c}{ Preoperative } & & \multicolumn{3}{c}{ 1-year follow-up } \\
\cline { 2 - 3 } \cline { 7 - 8 } & Group 1 & Group 2 & Group 3 & & Group 1 & Group 2 & Group 3 \\
\hline ZQR & 59 & 61 & 60 & & 28 & 23 & 33 \\
VAS & 91 & 88 & 93 & & 12 & 11 & 18 \\
ODI & 61 & 65 & 63 & & 11 & 13 & 23 \\
\hline
\end{tabular}

Abbreviations: ODI, Oswestry Disability Index; VAS, Visual Analog Scale; ZCQ, Zurich Claudication Questionnaire.

2 presented significantly less lumbar disc recurrences compared with group $3(P<0.05)$ and better clinical outcome when compared with the same group $(P<0.01)$.

Overall, ZCQ, VAS and ODI score improved in all the groups at 1 -year follow-up without significant variation in the subsequent follow-up (Figure 1). Significant statistical differences were noted in all the groups when comparing the clinical outcome measures from baseline to 1-year follow-up. A better clinical outcome was observed in group 2 when compared with group $3(P<0.01)$. Finally, recurrence of LDH was significantly less observed in group 2 (three patients) compared with group 3 (10 patients; $P<0.05$ ).

The mean preoperative and postoperative ZCQ, VAS and ODI scores are reported in Table 2.

\section{DISCUSSION}

In the current era, treatment of degenerative spine disease encompasses decompression of the neural elements and fusion, with or without instrumentation. Increasing in the understanding of spinal biomechanics, proliferation of sophisticated spinal devices, advances in bone fusion techniques, refinement of surgical approaches to the spine and the development of microsurgical and minimally invasive methods have made it possible to successfully treat several pathologies of the spine.

When we examine the issue of posterior spinal disease and LSS, it is well known that it should be considered both the natural history of the disease process and the iatrogenic instability resulting from a surgical decompression. As in a large majority of these patients, the symptoms encompass from radicular to central canal compression, they can require decompression of the paramedian lamina and at least the medial third or half of the facet complex. This, often, is also associated with microdiscectomy. Progressive resection of these structures can lead to progressive spinal instability. ${ }^{4,6}$ On the other hand, it well known that many patients with LSS, with or without grade I spondylolisthesis, do well without fusion and do not go on to have gross spinal instability after decompressive surgery. ${ }^{7}$ Accordingly, a motion-preserving technology that can be placed via a standard posterior approach can help to avoid fusion in many patients with stenosis in whom the spine is either only mildly unstable preoperatively or is made unstable after surgical decompressive destabilization of the facet complex. In this scenario, several devices designed for minimally invasive posterior stabilization have been introduced. Among these, the interspinous systems have been found able to provide local kyphosis, improve sagittal balance and segmental stability, modify posterior neural foraminal height, provide pain relief, particularly in patients suffering from low back pain due to facet overloading or segmental instability or increase lumbar spinal canal diameter in patients affected by LSS. $^{8-10}$ Furthermore, due to their segmental properties, it has been thought 
to have a role in maintaining lumbar intervertebral height following discectomy. ${ }^{11}$

In this paper, we retrospectively reviewed patient data collected over 6 years, in order to analyze the properties of interspinous devices in patients with LLS and LDH in whom the interspinous system has been inserted following microdiscectomy. Six years following surgical treatment, $85 \%$ of the patients of group 1 presented a very good improvement of symptoms and $90 \%$ of patients referred satisfaction for surgery. Only in few cases, reoperation was needed to change the implant. In one case, the device was removed and in two cases we observed the recurrence of symptoms and thus we changed the surgical strategy. Overall, patients of group 2 presented significantly less lumbar disc recurrences compared with group $3(P<0.05)$ and better clinical outcome when compared with the same group $(P<0.01)$. No significant complications were associated with such a kind of surgery. In particular, interspinous systems showed significant and clinically meaningful improvements in pain and disability since the first year post surgery for up to 6 years. Furthermore, interspinous devices have shown better clinical outcomes and less lumbar disc recurrences when associated with standard microdiscectomy. Our findings are in agreement with those of the previous studies that in shorter follow-up have shown the safety of the interspinous devices and suggested their ability in reducing the recurrent disc herniation rates. ${ }^{11,12}$ The latter issue, however, needs further evaluation as data provided so far are not completely concluding.

The concept that an implant placed between the spinous processes might provide relief for patients suffering from neurogenic intermittent claudication comes from some clinical studies and from the knowledge that most of these patients get relief from symptoms when they bend forward and flex their spines and conversely their symptoms worsen when they stand erect and extend their spines. ${ }^{13-17}$ Results from randomized, multicenter trials clearly demonstrate that the interspinous device improves clinical symptoms and function significantly compared with epidural steroid injections and conservative therapy in patients with symptoms of neurogenic intermittent claudication. ${ }^{18,19}$ Our findings are in agreement with these data and further provide the opportunity to compare patients with $\mathrm{LDH}$ in whom the interspinous system has been inserted following microdiscectomy with a control group. In comparison with other studies, we did not have a high rate of reoperation in the interspinous process device group. ${ }^{17,20}$ The results of this study lead to the overall conclusion that LLS treated with interspinous device is a safe treatment option to classic bony decompression. The use of the interspinous system, however, does not preclude subsequent decompressive surgery if required. Furthermore, we provided the evidence that the use of interspinous device following microdiscectomy can be of help in maintaining lumbar intervertebral height and reducing the rate of lumbar disc recurrences.

In the so-called 'minimally invasive surgery', interspinous devices have gained in importance and popularity quickly. What we have learned is that ISD can be used with beneficial effects in selected patients. But also that, similar to other novel technologies and techniques in spine surgery developed in recent decades, the early optimism for ISD has since waned significantly as a result in exceeding indication thus causing unfavorable outcomes. Although retrospective, our findings add new insight into the pertinent literature especially for the long follow-up. ISD may be considered as an alternative treatment to laminectomy for LSS, its effectiveness compared with laminectomy is unknown and a direct comparison between the two procedures in a prospectively multicenter randomized controlled study is needed to address this uncertainty.

\section{DATA ARCHIVING}

There were no data to deposit.

\section{CONFLICT OF INTEREST}

The authors declare no conflict of interest.

1 Fraser JF, Huang RC, Girardi FP, Cammisa FP Jr. Pathogenesis, presentation, and treatment of lumbar spinal stenosis associated with coronal or sagittal spinal deformities. Neurosurg Focus 2003; 14: e6.

2 Malmivaara A, Slatis P, Heliovaara M, Sainio P, Kinnunen H, Kankare J et al. Surgical or nonoperative treatment for lumbar spinal stenosis? A randomized controlled trial. Spine (Phila Pa 1976) 2007; 32: 1-8.

3 Weinstein JN, Tosteson TD, Lurie JD, Tosteson AN, Blood E, Hanscom B et al. Surgical versus nonsurgical therapy for lumbar spinal stenosis. N Engl J Med 2008; 358: 794-810.

4 Airaksinen O, Herno A, Kaukanen E, Saari T, Sihvonen T, Suomalainen O. Density of lumbar muscles 4 years after decompressive spinal surgery. Eur Spine J 1996; 5: 193-197.

5 Fox MW, Onofrio BM, Onofrio BM, Hanssen AD. Clinical outcomes and radiological instability following decompressive lumbar laminectomy for degenerative spinal stenosis: a comparison of patients undergoing concomitant arthrodesis versus decompression alone. J Neurosurg 1996; 85: 793-802.

6 Yone K, Sakou T, Kawauchi Y, Yamaguchi M, Yanase M. Indication of fusion for lumbar spinal stenosis in elderly patients and its significance. Spine (Phila Pa 1976) 1996; 21: 242-248.

7 Kim S, Mortaz Hedjri S, Coyte PC, Rampersaud YR. Cost-utility of lumbar decompression with or without fusion for patients with symptomatic degenerative lumbar spondylolisthesis. Spine J 2012; 12: 44-54.

8 Anderson PA, Tribus CB, Kitchel SH. Treatment of neurogenic claudication by interspinous decompression: application of the X STOP device in patients with lumbar degenerative spondylolisthesis. J Neurosurg Spine 2006; 4: 463-471.

9 Alfieri A, Gazzeri R, Prell J, Scheller C, Rachinger J, Strauss C et al. Role of lumbar interspinous distraction on the neural elements. Neurosurg Rev 2012; 35: 477-484.

10 Kim KA, McDonald M, Pik JH, Khoueir P, Wang MY. Dynamic intraspinous spacer technology for posterior stabilization: case-control study on the safety, sagittal angulation, and pain outcome at 1-year follow-up evaluation. Neurosurg Focus 2007; 22: E7.

11 Caserta S, La Maida GA, Misaggi B, Peroni D, Pietrabissa R, Raimondi MT et al. Elastic stabilization alone or combined with rigid fusion in spinal surgery: a biomechanical study and clinical experience based on 82 cases. Eur Spine J 2002; 11 (Suppl 2), S192-S197.

12 Patil S, Burton M, Storey C, Glenn C, Marino A, Nanda A. Evaluation of interspinous process distraction device (X-STOP) in a representative patient cohort. World Neurosurg 2013; 80: 213-217.

13 Zucherman JF, Hsu KY, Hartjen CA, Mehalic TF, Implicito DA, Martin MJ et al. A multicenter, prospective, randomized trial evaluating the $X$ STOP interspinous process decompression system for the treatment of neurogenic intermittent claudication: two-year follow-up results. Spine (Phila Pa 1976) 2005; 30: 1351-1358.

14 Miller LE, Block JE. Interspinous spacer implant in patients with lumbar spinal stenosis: preliminary results of a multicenter, randomized, controlled trial. Pain Res Treat 2012; 2012: 823509.

15 Davis RJ, Errico TJ, Bae H, Auerbach JD. Decompression and Coflex interlaminar stabilization compared with decompression and instrumented spinal fusion for spinal stenosis and low-grade degenerative spondylolisthesis: two-year results from the prospective, randomized, multicenter, Food and Drug Administration Investigational Device Exemption trial. Spine (Phila Pa 1976) 2013; 38: 1529-1539.

16 Hobart J, Gilkes C, Adams W, Germon T. Interspinous spacers for lumbar foraminal stenosis: formal trials are justified. Eur Spine J 2013; 22 (Suppl 1), S47-S53.

17 Stromqvist BH, Berg S, Gerdhem P, Johnsson R, Moller A, Sahlstrand T et al. X-stop versus decompressive surgery for lumbar neurogenic intermittent claudication: randomized controlled trial with 2-year follow-up. Spine (Phila Pa 1976) 2013; 38: $1436-1442$

18 Kovacs FM, Urrutia G, Alarcon JD. Surgery versus conservative treatment for symptomatic lumbar spinal stenosis: a systematic review of randomized controlled trials. Spine (Phila Pa 1976) 2011; 36: E1335-E1351.

19 Moojen WA, Arts MP, Bartels RH, Jacobs WC, Peul WC. Effectiveness of interspinous implant surgery in patients with intermittent neurogenic claudication: a systematic review and meta-analysis. Eur Spine J 2011; 20: 1596-1606.

20 Moojen WA, Arts MP, Jacobs WC, van Zwet EW, van den Akker-van Marle ME, Koes BW et al. Interspinous process device versus standard conventional surgical decompression for lumbar spinal stenosis: randomized controlled trial. BMJ 2013; 347: f6415. 